\title{
离 Una escuela hecha por todos
}

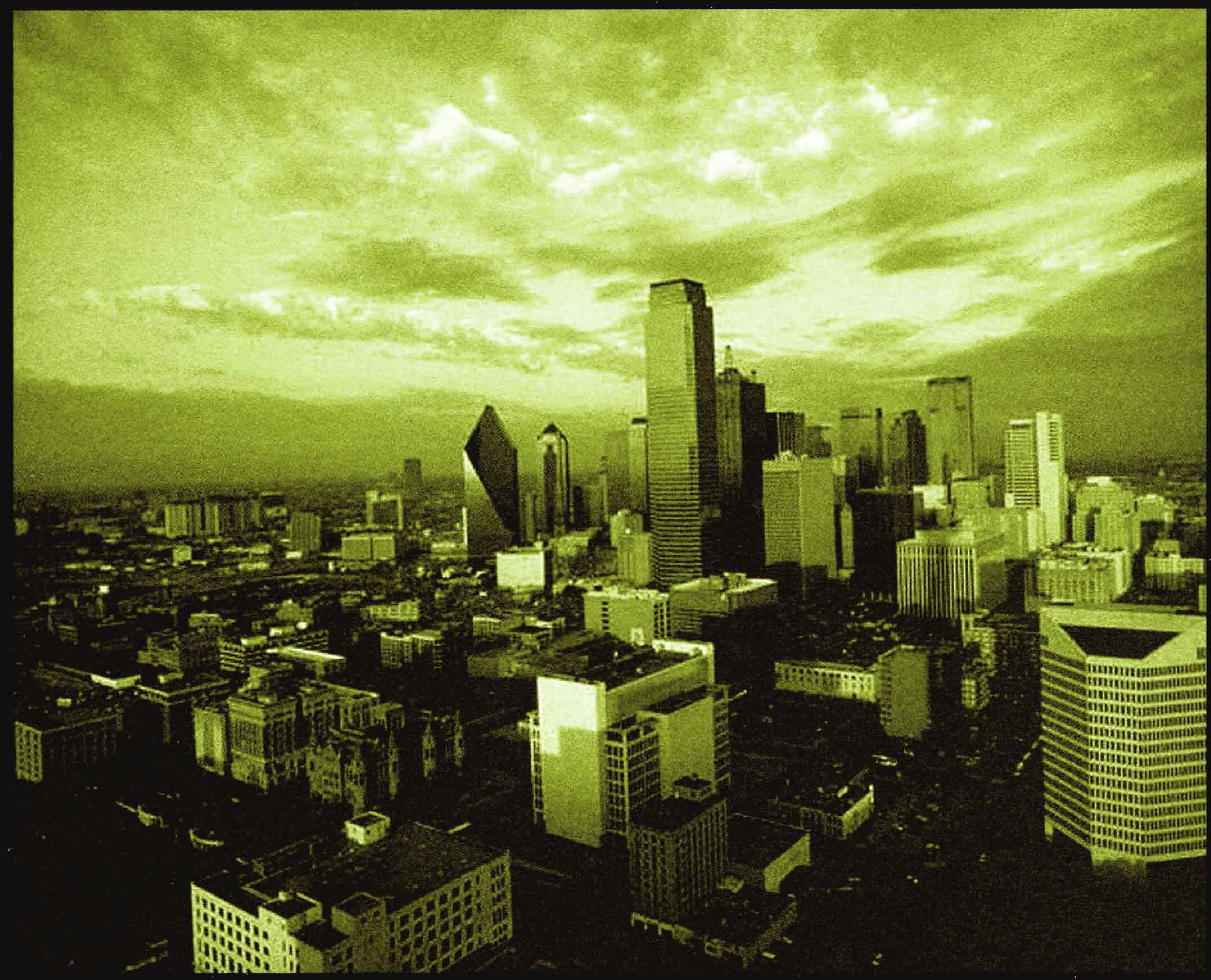




\section{Maria do Pilar Lacerda Almeida e Silva}

Graduada en Historia por la Universidad Federal de Minas Gerais. 2001, especialización en Gestión de Sistemas Educativos, por la Pontifícia Universidade Católica de Minas Gerais 1993 a 1996, Directora del Centro de Capacitación de los Profesionales de la Educación de la Municipalidad de Belo Horizonte. 1999 a 2001, Consultora del Ministerio de la Educación en el Programa de Capacitación de los Secretarios Municipales de Educación. 2002, gerente de la Información de la Secretaría de las Politicas Sociales de la Municipalidad de Belo Horizonte. Desde 2002 Secretaria de la Educación de la Municipalidad de Belo Horizonte y 2005 Presidente Brasileña de la Unión de los Dirigentes Municipais de Educación- UNDIME. Publicaciones: Escuela pública para todos: ¿dónde está la contradicción?

\section{Áurea Regina Damasceno}

Asesora Pedagógica del Gabinete de la Secretaría de Educación de Belo Horizonte.

\section{Tadeu Rodrigo Ribeiro}

Asesor Pedagógico del Gabinete de la Secretaría de Educación de Belo Horizonte.

\section{Resumen}

Este artículo recoge la experiencia de la Escuela Plural en Belo Horizonte, Brasil, la cual está inscrita en los paradigmas republicanos como la democracia, la participación y el reconocimiento de los derechos de los sujetos. La inclusión social y la educación para todos, se articulan a un proyecto político pedagógico de largo alcance para el desarrollo de la ciudad mediante proyectos que involucran a toda la comunidad.

\section{Palabras clave}

Escuela plural, paradigma republicano, inclusión, proyecto político pedagógico.

\section{Abstract}

This article summarizes the experience of the Plural School in Belo Horizonte, Brazil, which is inserted in some republican paradigms such as democracy, participation, and the recognition of people rights. The social inclusion and education for everybody gather in a pedagogic and political program that pretends to help the city development through a variety of projects that involve the whole community.

\section{Key words}

Plural School, republican paradigm, inclusion, pedagogic and political program. 
II

os debates sobre cómo garantizar el derecho a una educación de calidad se centra en cómo hacer una Escuela pública de verdad. No tenemos en nuestro país una historia de la escuela para todos. Las escuelas públicas eran para pocos niños y niñas. Hasta los años 80 , apenas el $80 \%$ de los niños y niñas tenían acceso. Y quienes la frecuentaban, enfrentaban mecanismos de exclusión como la reprobación.

Brasil, así como todos los países de América Latina, sufre con la concentración de renta que origina desigualdades sociales. Como tenemos sociedades muy heterogéneas, es necesario tener esto en cuenta cuando pensamos los proyectos pedagógicos y el trabajo, por eso las secretarías de educación deben tener un foco muy grande, claro y una gestión pedagógica.

En Brasil tenemos que enfrentar dos desafíos: hacer la escuela para todos y hacer una escuela donde todos aprendan, garantizar el acceso, la permanencia y el aprendizaje. Una escuela pública hecha por todos y para todos tiene que ser republicana, orientada por una pedagogía libertadora. Pero, ¿por qué una escuela republicana y una pedagogia libertadora?

Los paradigmas republicanos -la democracia, la participación y el reconocimiento de los derechos de los sujetos- tienen que traspasar las relaciones entre las instituciones que dirigen las políticas económicas y públicas, entre los actores pedagógicos, entre estos y los niños y las niñas, así como entre los padres y las madres.

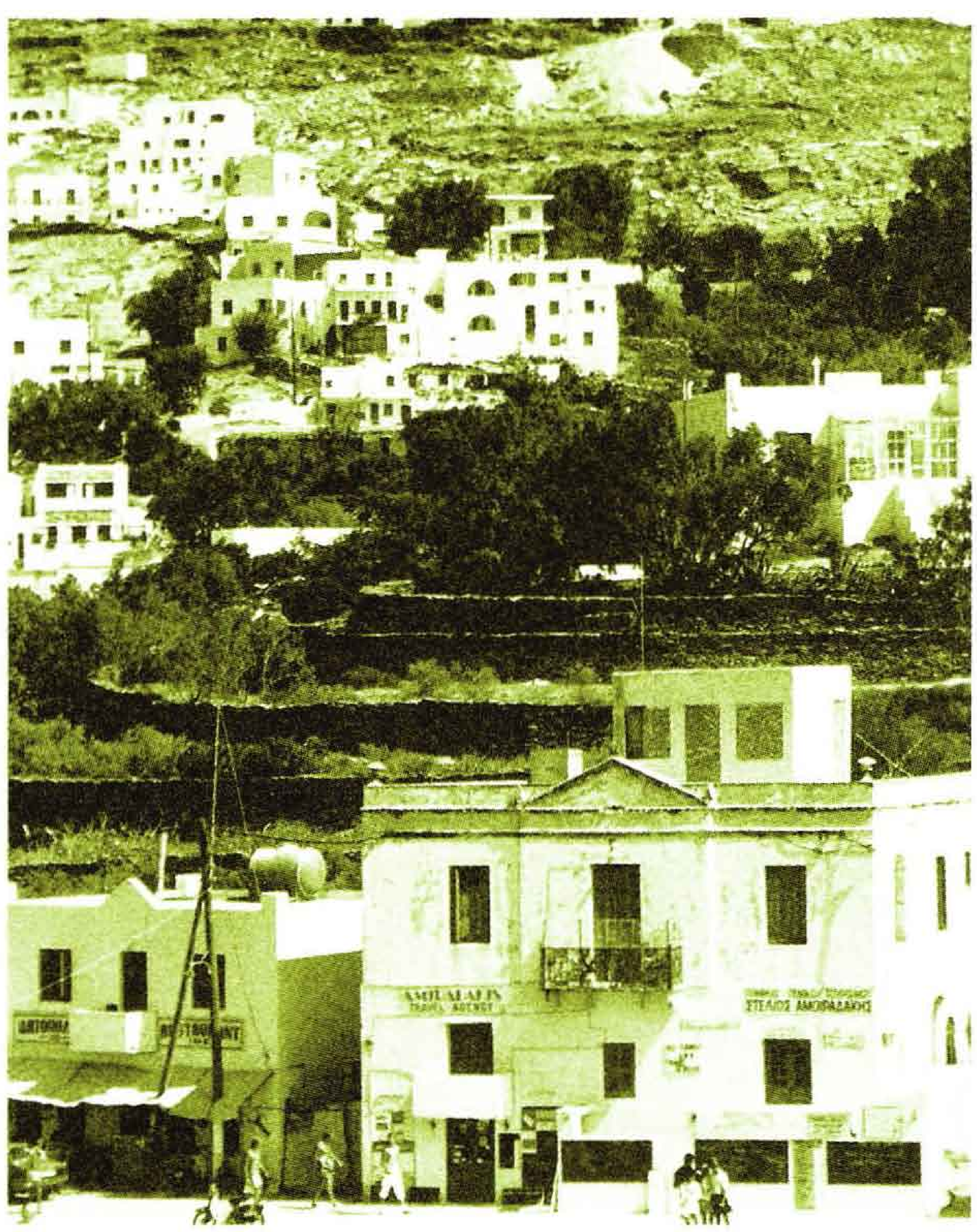

La pedagogía no es neutra, no está apartada de la sociedad, la pedagogía es política. Es necesario que pensemos en cómo hacer un proyecto político pedagógico que efectivamente libere a niños, niñas y jóvenes de la explotación y de la pobreza extrema, situaciones a veces indignas e inhumanas que se viven en Brasil y en Latinoamérica.

La pedagogía libertadora concibe la formación integral del aprendiz en las dimensiones humana, política, cultural y cognitiva en la búsqueda de la igualdad social, la inclusión y la convivencia pacífica entre quienes son diferentes.

Lo cotidiano en la escuela es muy importante. No podemos hacer una política administrativa escolar que no se ligue a lo cotidiano y a la realidad de las escuelas que son tan diferentes y variadas. Un pro- 
yecto político pedagógico que efectivamente tenga un carácter republicano, debe tener en cuenta que hay varios protagonistas en la escuela hoy, no son sólo los maestros y las maestras, sino también los alumnos, los padres, las madres y los líderes comunitarios. Todos los miembros de la comunidad deben participar de la elaboración, desarrollo y evaluación del proyecto de cada escuela. Así, debemos tener en cuenta que hay, en principio, dos concepciones de proyeto político pedagógico.

La primera, sólo tiene como resultado los números: número de escuelas construidas, número de matrículas en las escuelas, número de reprobaciones, número de maestras y maestros y valor del presupuesto invertido en educación. Los niños y las niñas son apenas números, no sujetos de derechos, activos y políticos. En Brasil, esta concepción de proyecto pedagógico orientó los gobiernos neoliberales en los últimos 10 ó 15 años. Los ministerios y las secretarías de educación enfocaron los resultados de evaluaciones externas y no se detuvieron a mirar la escuela como espacio público de formación de sujetos. La escuela fue considerada como una gran empresa, y en ese sentido los niños y las niñas fueron concebidos como productos..

Muchos años han transcurrido desde que las administraciones públicas encargadas de la educación empezaran a comprender que una buena politica educativa que garantice el derecho a la educación no pasa sólo por la creación de escuelas, sino también por el reconocimiento de la diversidad cultural 
de los aprendices. Pero requería de la creación de políticas capaces de garantizar el acceso, la permanencia y el aprendizaje con éxito.

Hoy la realidad y los debates en Brasil demuestran que las administraciones progresistas, democráticas y populares mantienen una concepción de proyeto politico pedagógico basada en el sujeto de derechos; es decir, en el derecho al acceso, permanencia y aprendizaje en la escuela. Cada decisión política tiene en cuenta cómo incidirá sobre un niño o una niña, personas de verdad, para las cuales la escuela debe ser pensada. Una política social que intente la inclusión necesita de otras políticas sociales que la sostengan. En el caso de una política educativa, hay que desarollar programas y acciones que garanticen el acceso, la permanencia y el aprendizaje con éxito.

Para sostener la permanencia, hay que desarrollar acciones que saquen a los niños y a las niñas del trabajo infantil de las metrópolis y garanticen el derecho de ser niños y a frecuentar la escuela, políticas de acompañamiento socioeducativo de los grupos familiares, distribución a los alumnos de conjuntos escolares compuestos por items adecuados a cada edad, así como programas de alimentación en la escuela.

Para mejorar la planificación de la oferta de plazas en las redes de enseñanza, es necesario identificar la demanda. Este trabajo democratiza el acceso y asegura el derecho del alumno a estudiar próximo a su domicilio.
El aprendizaje merece una gran inversión en lo que respecta a la organización del trabajo escolar, a la formación de los maestros y al estudio y análisis de sus prácticas. Por otro lado, el proceso de evaluación del aprendizaje y del proceso educativo requiere de una particular atención. La creación de bibliotecas como espacios de lectura, investigación y ocio y la inclusión digital de alumnos, fucionarios y comunidad por medio de la instalación de equipos conectados a la Internet banda ancha, son programas pedagógicos fundamentales para el éxito escolar, pues proveen a los alumnos de condiciones para aprender a aprender y a seguir aprendiendo por sí mismos.

Las políticas educativas no pueden olvidar la formación continuada en el trabajo de los maestros y las maestras. Hay que crear una red de formación en la cual los maestros puedan estudiar, reflexionar sobre sus prácticas y cambiar gracias a la experiencia.

No se puede separar la práctica escolar de la investigación y debemos preocuparnos por formar docentes reflexivos que tengan tiempo escolar, tiempo en sus jornadas de trabajo para que puedan estudiar reflexionar y construir juntos las propuestas del proyecto pedagógico de cada escuela. Asimismo, la política de formación debe comprender también una carrera de magisterio con evaluación de desempeño e incentivo a la continuidad de estudios en graduación y posgrado.

La gestión democrática de la escuela tiene un papel fundamental en el desarrollo del proceso educativo, pues debe conducir los procesos de la organi- 
zación del trabajo y tiempos escolares, orientar la construcción del currículo y la participación de la comunidad en las decisiones. En otro nivel, es responsable de mantener la articulación con otras instituciones escolares, con las administraciones centrales de la educación y con las políticas sociales, ampliando la relación de la escuela con la ciudad.

Los proyectos políticos y pedagógicos orientados por los principios republicanos y por la pedagogía libertadora serán concretos si la ciudad, las provincias y el país efectivamente o asumen. Así, la inclusión social debe ser nuestro principio básico, nuestra concepción de escuela es una escuela para todos y donde todos aprendan como sujetos transformadores de su tiempo.

\section{Política pedagógica de Belo Horizonte La Escuela plural}

A finales de la década del 70, los profesores de la Red Municipal de Educación de Belo Horizonte se insertaron en el movimiento de redemocratización del país y en el movimiento de renovación pedagógica. Esto aumentaba sus avances en el reconocimiento y en la lucha por sus derechos. Este movimiento se reflejó sobre sus prácticas pedagógicas. Los profesores, con audacia y profesionalismo, proponían y desarrollaban experiencias significativas en las escuelas. Estas experiencias supusieron la búsqueda de respuestas educativas a la desigualdad social y a los procesos de exclusión presentes en las escuelas, señalaban una concepción de educación diferente

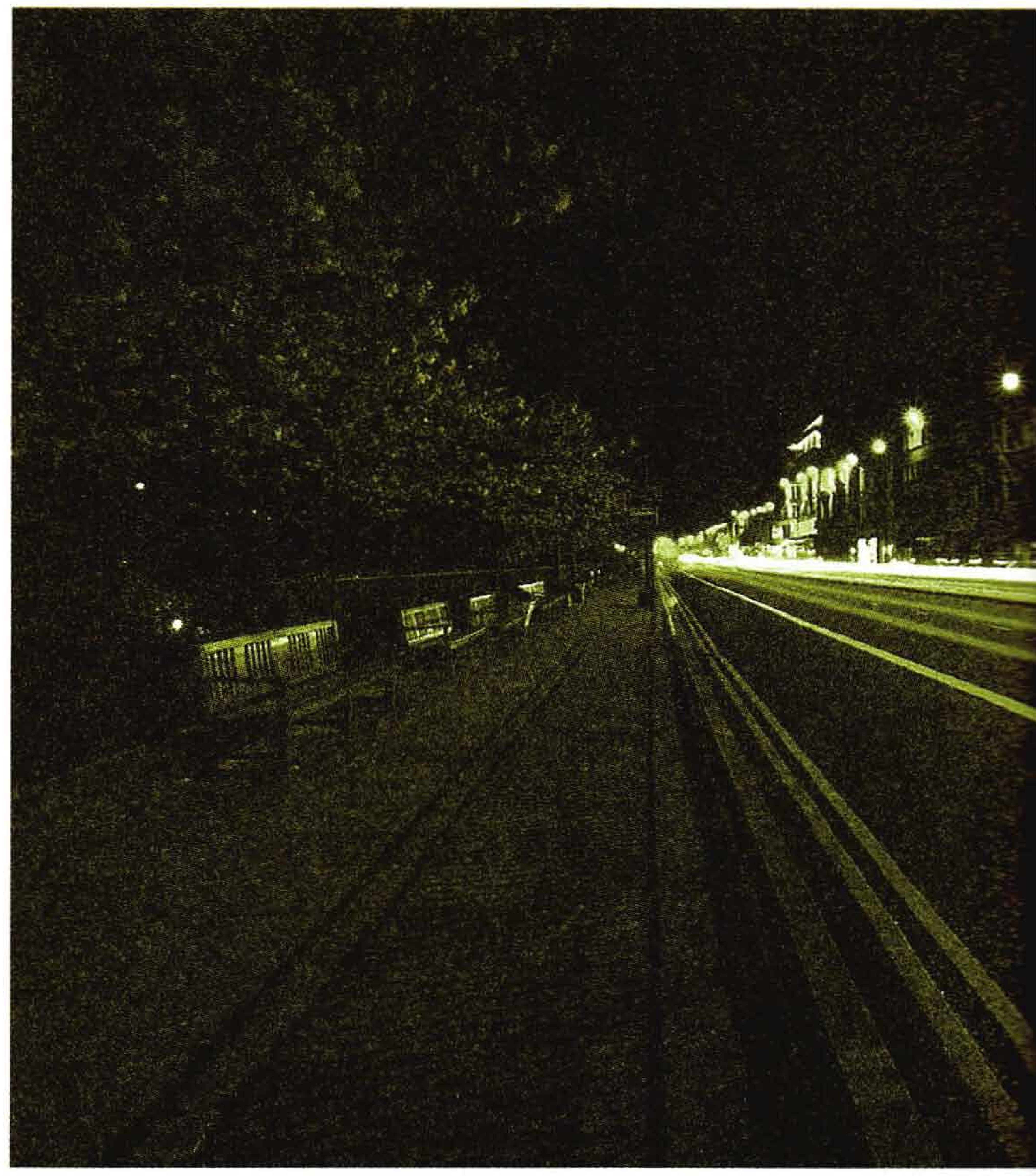

del modelo culturalmente aceptado, así como direcciones innovadoras para la educación proponiendo cambios en las escuelas que se reflejaran en el aprendizaje de los alumnos.

Como los cambios propuestos no eran reconocidos por la legislación, en 1995, el gobierno democrático popular que asumió el poder tuvo la sensibilidad política y el coraje de sistematizar las experiencias desarrolladas por los profesores en las escuelas municipales y de devolverlas como una propuesta de política pedagógica para la Red Municipal de Educación. Nació, así, la Escuela plural, el cual, como proyecto político-pedagógico innovador, en diez años impulsionó cambios basados en el respeto a la diver- 


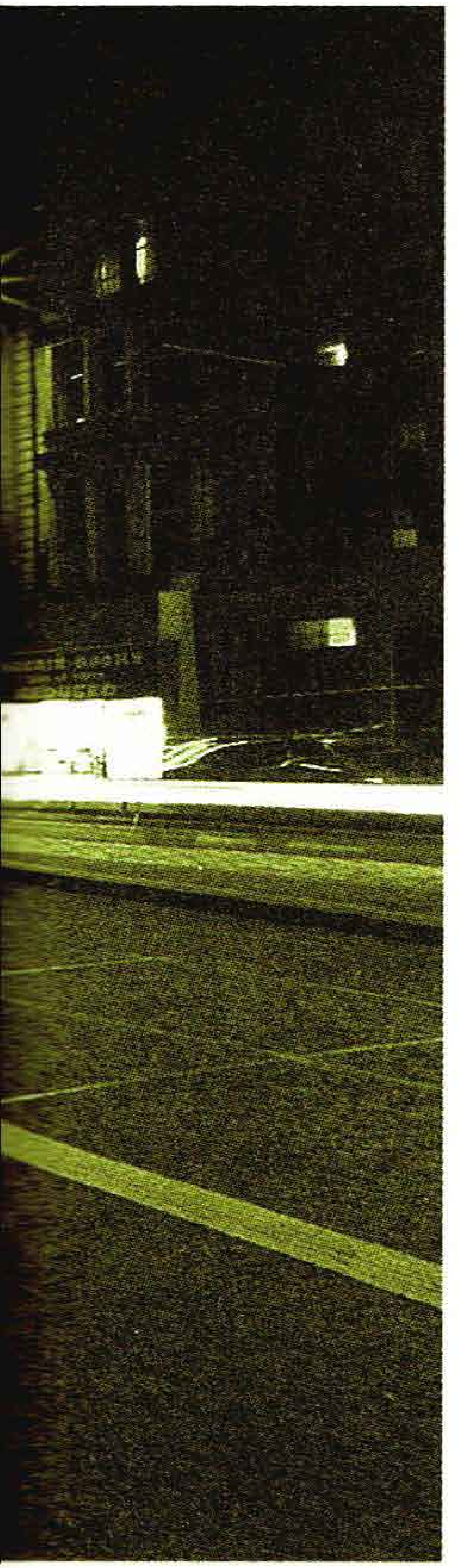

sidad, a los sujetos y a sus saberes y quehaceres, así como a la valoración y cualificación permanente de los maestros y de las maestras.

La Escuela plural fue implantada en 1995 en las escuelas municipales de Belo Horizonte con el objetivo de garantizar el derecho a la educación para todos teniendo como principio la inclusión social. La enseñanza se organiza en ciclos de edad de formación: $1^{\text {er }}$ ciclo ( 6 a 9 años), $2^{\circ}$ ciclo ( 9 a 12 años), $3^{\text {er }}$ ciclo (12 a 15 años), $4^{\circ}$ ciclo o Enseñanza Media.

Belo Horizonte salió adelante. Fue la primera ciudad del país que recibió a niños de 6 años en la Enseñanza Fundamental ${ }^{1}$. El tiempo escolar es flexible, respeta los ritmos diferenciados de aprendizaje de los alumnos, y la formación del profesor está orientada hacia la reflexión sobre la práctica pedagógica y la interacción con el alumno y su familia. La comunidad participa de la gestión democrática de la escuela.

En diez años, la Escuela plural vivió un tiempo de cambios en el sentido de construir una escuela orientada por el respeto a la diversidad, a los sujetos y a

1 En Brasil, la educación se organiza en: Educación Superior y Educación Básica. La Básica se organiza en 11 años de estudios: Educación Infantil: 0 a 5 años y 8 meses; Ensenãnza Fundamental: 6 años a 14 ó 15 años -estudios realizados durante 9 años-, y Ensenãnza Media: fin de la Educación Básica -estudios realizados durante 3 años-. su saber-hacer y por la construcción colectiva. En ese trayecto, muchos avances fueron alcanzados: hoy la escuela municipal de Belo Horizonte se ha hecho mucho más inclusiva y abierta a las comunidades, más democrática y materialmente mejor estructurada.

\section{La Escuela plural ejes orientadores}

1 Intervención en la estructura de la escuela excluyente, garantizando el acceso y la permanencia del alumno, con calidad.

2 Formación humana en su totalidad: los alumnos son sujetos socioculturales con múltiples potencialidades.

3 Escuela como espacio de vivencia cultural.

4 Escuela como experiencia de producción colectiva.

5 Niños y adolescentes como sujetos de derecho en el presente.

6 Socializaciọ́n adecuada a cada ciclo de edad de formación.

7 Nueva identidad de la escuela y de sus profesionales.

\section{Programas desarrollados}

\section{Registro escolar}

Trabajo conjunto de las Secretarías de Educación del Estado y del Municipio. El Registro escolar de Belo Horizonte fue creado en 1974 con el objeti- 
vo de identificar la demanda desde los 6 años de edad, para ingreso en la Enseñanza Fundamental. Esa demanda permite la planificación de la oferta de plazas en las dos redes.

Desde 1993 la inscripción en el Registro Escolar se hace en el Correo con la presentación de la factura de energía. El identificador de la factura actúa como referencia para el geo-procesamiento, el cual permite que los alumnos estudien cerca de sus residencias. Ese sistema elimina colas y democratiza el acceso a la Red Pública de Enseñanza. Anualmente, cerca de 30 mil candidatos son encaminados a las escuelas estatuales y municipales de Enseñanza Fundamental en Belo Horizonte. El Registro Escolar fue premiado en 2001 por la Fundación Ford, como uno de los 30 mejores programas de políticas sociales del país.

\section{Política de acompañamiento sistemático por microrregiones}

Esta política estratégica permite el diagnóstico y constucción de directrices político pedagógicas para la Red Municipal de Educación. El acompañamiento por micorregión evalúa el desarollo y el éxito de acciones que involucran la práctica escolar, la organización interna y la elaboración curricular, la ejecución de proyectos, la relación profesor-alumno y la implicación de la escuela con la comunidad.

Por el sistema, cada regional administrativa de Belo Horizonte es dividida en microrregiones que incluyen, máximo, cuatro escuelas posibilitando su dinamismo y un acompañamiento próximo al cotidiano escolar.

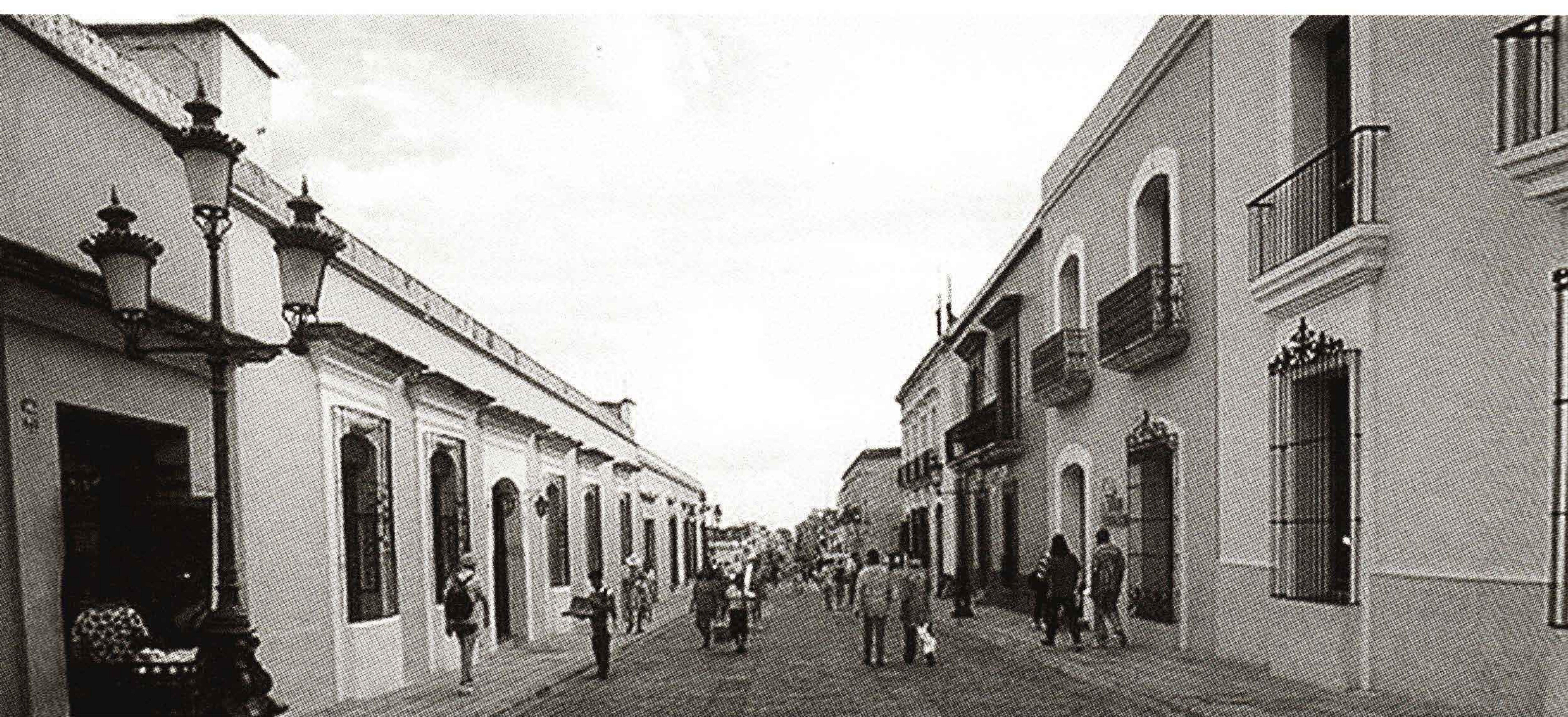




\section{Familia-Escuela}

El proyecto busca estimular la presencia de la familia en la vida escolar. Es una propuesta permanente de comunicación directa con los familiares de los niños, de los adolescentes y de jóvenes de la Red Municipal de Educación. Cuenta con acciones como encuentros, foros, seminarios y conferencias y cuenta con un servicio telefónico público de atención al ciudadano.

\section{BEM-BH}

El programa Beca-Escuela Municipal de Belo Horizonte, BEM-BH, fue creado en 1996. Es una iniciativa pionera en Brasil y se considera una referencia en programas de transferencia de recursos con fines educativos.

Además de repasar el beneficio financiero, el BEM-BH hace acompañamiento socio-educativo a los grupos involucrados a través de reuniones, visitas domiciliarias y cursos de alfabetización de adultos y de formación profesional. Asimismo colabora en del Programa Beca-Familia acompañando la frecuencia escolar de los alumnos beneficiados por el programa federal.

\section{Programa de Evaluación de Aprendizaje}

La Red Municipal de Educación de Belo Horizonte participó, en 2002, del Sistema de Minas Gerais de Evaluación de la Escuela Pública. Fueron eva- luadas las capacidades en idioma portugués, en el área de lectura. En 2003, también fueron evaluadas las capacidades en matemática. Las dos evaluaciones incluyeron alumnos de la mitad y del final de la Enseñanza Fundamental y de alumnos que estaban concluyendo la Enseñanza Media.

Para la mejora de la calidad de la enseñanza, el gobierno municipal repasó, durante 2003 y 2004, un monto extra de $\mathrm{R} \$ 16$ millones para mantenimiento y desarrollo de proyectos, cuya aplicación cuenta con la participación de la comunidad.

\section{Biblioteca escolar y Programa de lectura}

Desde 1997 se implantó el Programa de Bibliotecas de la Red Municipal de Educación con el nombramiento de bibliotecarios y auxiliares de bibliotecas. El Programa está estructurado en bibliotecas-polo, que tienen por finalidad atender a la comunidad escolar y a la comunidåd situada en el entorno de la escuela, además de coordinar el trabajo de bibliotecas escolares a ellas agregadas. Las bibliotecas forman parte del proyecto pedagógico de las escuelas y son espacios de lectura, recreación e investigación. Las 181 bibliotecas escolares poseen acervo diversificado y actualizado, porque el 10\% de las subvenciones municipales enviadas a las cajas escolares son aplicadas al acervo. En 2004 fueron adquiridos 200 mil libros para las bibliotecas, incluyendo ediciones para el profesor y 56 títulos de literatura afro-brasileña. 

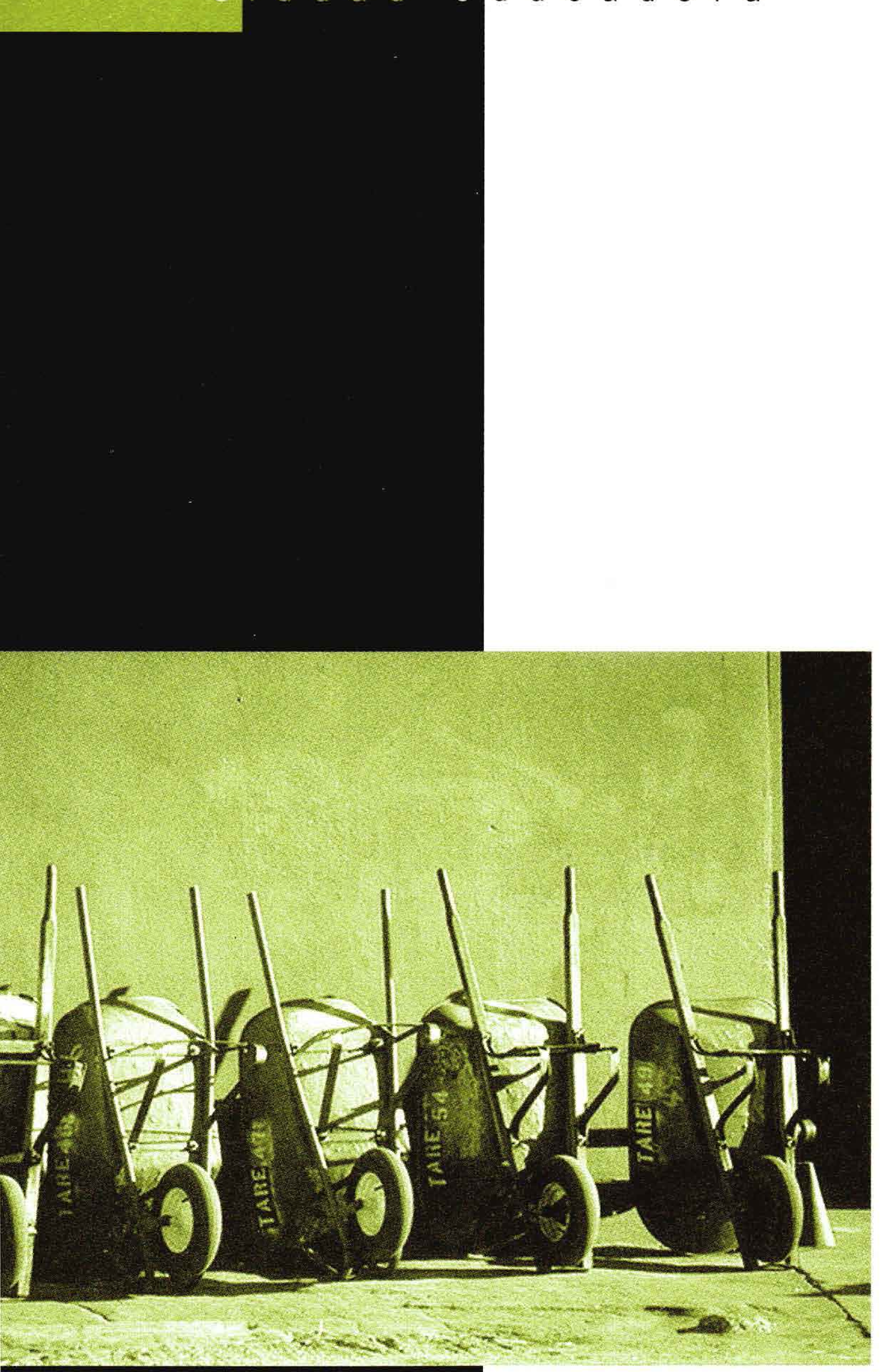

Fueron adquiridos, para los alumnos de la Enseñanza Fundamental, Educación Infantil y Guarderías asociadas, libros de literatura de diferentes géneros y temas. Paralelo a esas adquisiciones, se están desarrollando acciones apuntando a incentivar la lectura y la formación del alumno y del profesor lector.

\section{Inclusión de alumnos con minusvalía}

La inclusión escolar en el gobierno municipal de Belo Horizonte ha sido construida por medio de acciones pedagógicas comprometidas con el derecho a la educación. Esas acciones buscan garantizar el acceso y la permanencia de alumnos con minusvalías en escuelas regulares de la Red Municipal de Educación, respetando principios fundamentales de una escuela pública, plural e inclusiva.

\section{Estrategias y acciones}

- Prioridad de matrícula en la Educación Infantil para niños con deficiencia.

- Inversión en la formación de los profesionales de educación.

- Acompañamiento a las escuelas por el Equipo de apoyo a la inclusión de personas con deficiencia, conductas típicas y altas habilidades considerando alumnos, profesores y educadores de la Red Municipal de Educación y Guarderías asociadas. 
un a es c u el a h e c h a p o r t o d o
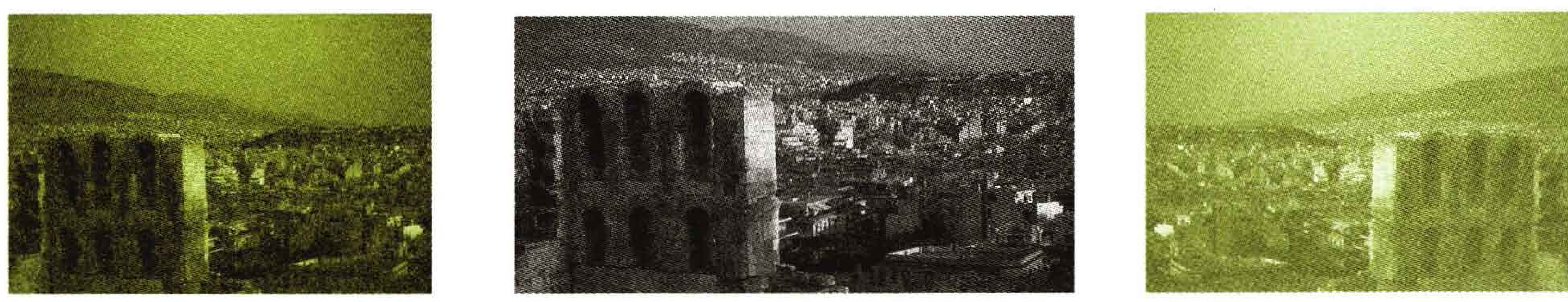

- Disponibilidad de equipos, recursos materiales y adecuación de la red física de las escuelas.

- Creación del Centro de Apoyo Pedagógico para Atención a las personas con deficiencia visual, CAP-BH, que ofrece trascripción en Braille, capacitación para uso de computadoras, software y producción de materiales.

- Proyecto de escolarización de sordos con profesores formados en LiBras, Lengua Brasileña de Señales.

- Intérpretes e instructores de Libras en la escuela.

- Colaboración de Red Sarah de Hospitales, Universidades y ONG.

- Articulación intersectorial entre Educación, Salud, Asistencia Social y demás organismos y sectores de la sociedad civil y del poder público.

\section{Promoción de la igualdad racial}

La Secretaría Municipal de Educación (SMED) creó en 2004 el Núcleo de relaciones étnico-raciales y de género, con el objetivo de establecer una política educacional que posibilitara la valoración de la diversidad y la superación de la desigualdad étnico-racial. Fueron elegidos, inicialmente, tres ejes de trabajo:
- Formación de profesores con la realización de congresos y seminarios, momentos permanentes de formación por medio de cursos, exposiciones y debates.

- Inversión en materiales didáctico-pedagógicos, como películas, documentales y libros para la Biblioteca del profesor, subvencionar el trabajo y la formación docente.

- Construcción de una política para la implementación de la Ley 10.639/03, que determina la obligatoriedad de la enseñanza de la historia de África y de los africanos en el currículo de la Educación Básica, la cual busca la valoración y el reconocimiento de la participación de la población negra en la construcción y formación de la sociedad brasileña.

La trayectoria de la inserción de la temática de las relaciones étnico-raciales en las escuelas municipales comenzó, sobre todo, con la formación docente. El programa Escuela plural es radical en su principio de una educación que pueda incluir a todos, sin distinción. El gobierno municipal de Belo Horizonte ha trabajado para que esa inclusión se realice de hecho. Fue ampliado el acervo de materiales didáctico-pedagógicos, los cuales abordan la temática racial; además se realizaron diversos congresos y seminarios, momentos 
permanentes de formación por medio de cursos, exposiciones y debates que han señalado algunos caminos. Es en ese contexto que se inserta la discusión de las relaciones raciales y la educación en la Red Municipal de Educación.

\section{Alfabetización y letramento}

La Secretaría Municipal de Educación, dentro del propósito de formular políticas para que la alfabetización sea garantizada para todos, creó un Núcleo de Alfabetización y Letradura. El Núcleo asesora a las escuelas de la Red Municipal de Educación en la formación de profesores y en el estudio y análisis de las prácticas de la alfabetización y de la letradura, e implantó un proyecto de emergencia para la alfabetización de jóvenes del $3^{\text {er }}$ ciclo. Las directrices de este trabajo son la relación enseñanza-aprendizaje, las interacciones entre profesores y alumnos, el tratamiento de la diversidad y organización del trabajo escolar, y la evaluación de la situación actual de la enseñanza y del aprendizaje

\section{Educación patrimonial, urbana y ambiental}

El gobierno municipal de Belo Horizonte, por intermedio del Núcleo Ciudad y Medio Ambiente, de la Secretaría Municipal de Educación, desarrolla proyectos que apuntan, sobre todo, a la educación para el cuidado y conservación del patrimonio urba- no y ambiental. Alumnos y profesores de la Red Municipal de Educación son motivados a reflexionar de manera continua de las cuestiones que involucran al patrimonio y ambiente urbano, investigando y pensando sobre los lugares de la ciudad, sus formas de ocupación y apropiación. Así, tienen la oportunidad de conocer más profundamente la historia de la ciudad y de las comunidades en las que viven y construir caminos para el ejercicio de la ciudadanía. Los trabajos, acompañados por orientadores, incluyen el levantamiento y registro de la memoria local, la elaboración de proyectos ambientales y la realización de exposiciones, incluso en el ámbito de la Muestra Plural. Los proyectos cuentan con la colaboración

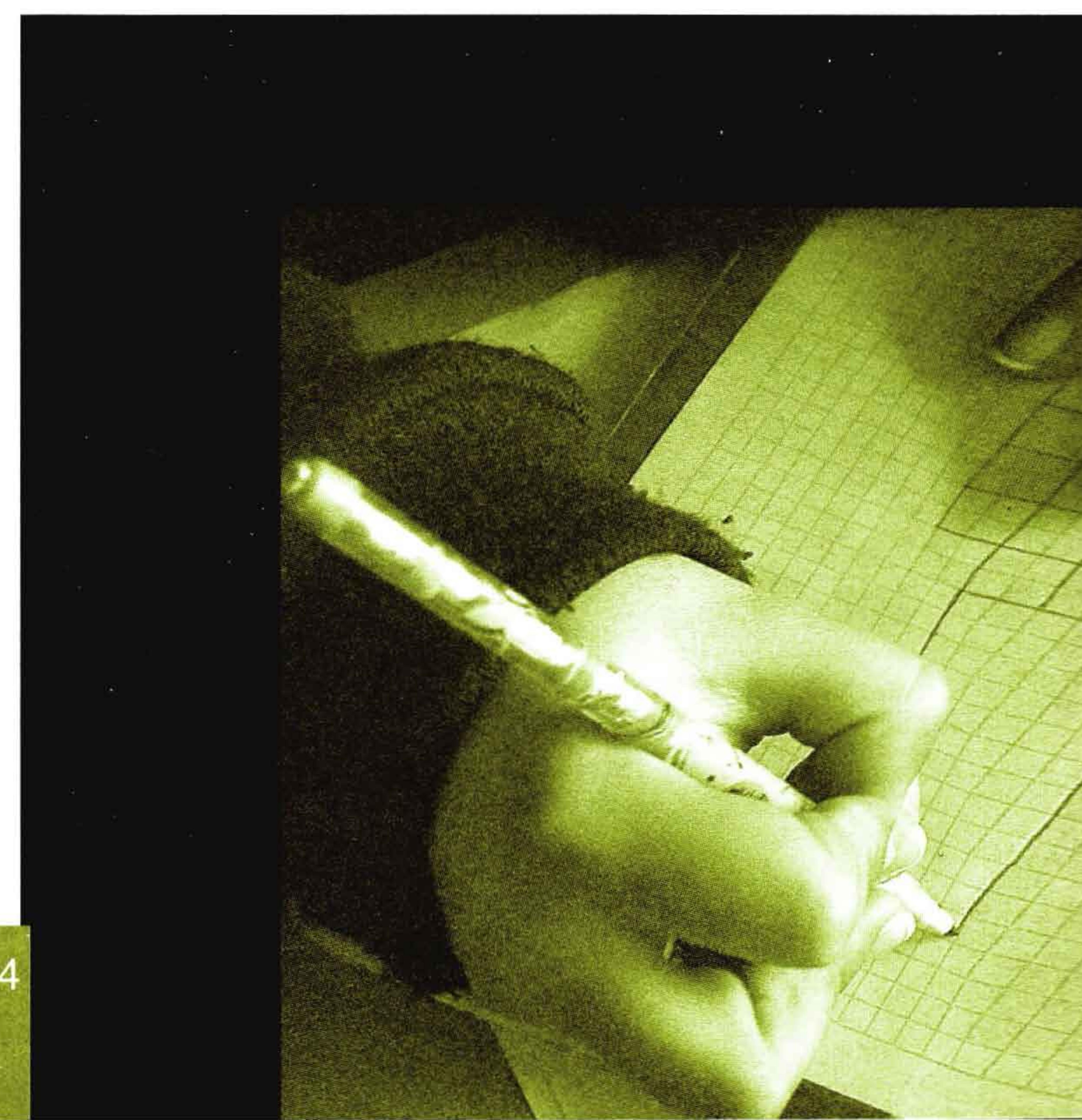


de otras Secretarías municipales, del Gobierno Federal, de la Universidad Federal de Minas GeraisUFMG, y de otras instituciones públicas y privadas.

\section{Educación infantil}

La ampliación de la oferta de Educación infantil, primera etapa de la Educación Básica, constituye uno de los hitos de la actual política de educación del municipio. La Secretaría Municipal de Educación de Belo Horizonte, además de implantar la atención a los niños de cero a tres años en tiempo integral, triplicó la atención a los niños de tres a cinco años y ocho meses, en tiempo parcial, en la Red

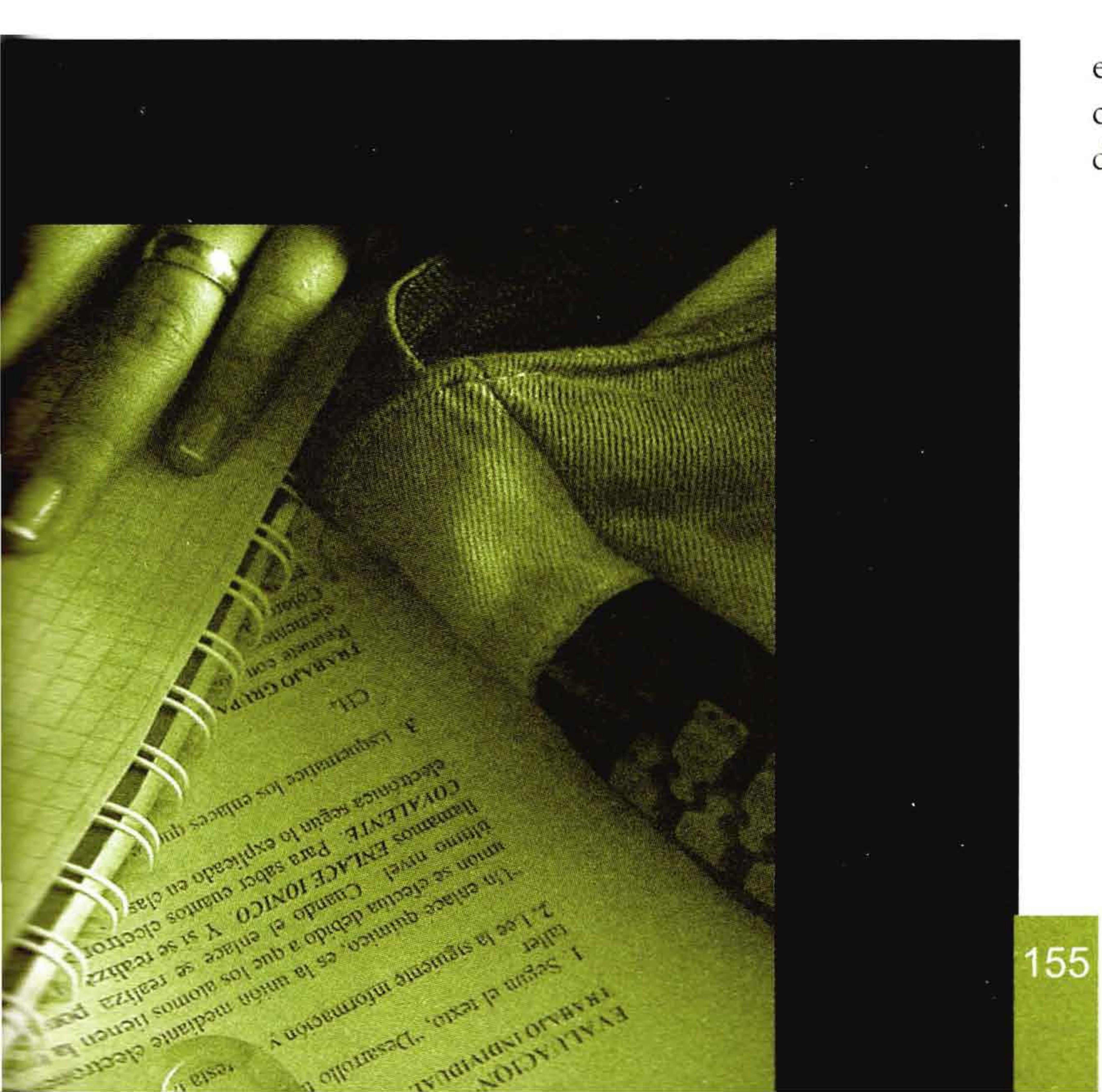

Municipal de Educación. Para la expansión de ese servicio, la Secretaría Municipal de Educación priorizó regiones de gran vulnerabilidad social.

Otra forma de actuación del municipio en el área de la Educación Infantil es por medio de convenios entre el gobierno municipal de Belo Horizonte y 195 instituciones comunitarias y filantrópicas, que atienden a niños entre cero y cinco años y ocho meses. Además de los recursos financieros, el gobierno municipal suministra alimentos, acompaña la salud de los niños y apoya la formación del cuerpo docente.

\section{$\mathrm{BH}$ sin analfabetos}

Con el objetivo y el compromiso de eliminar el analfabetismo y de ampliar la escolarización, la Secretaría Municipal de Educación actúa en 4 frentes de trabajo:

1 EJA - Ed̨ucación de Jóvenes y Adultos. Modalidad de enseñanza que atiende a jóvenes y adultos con más de 14 años, interesados en iniciar o continuar los estudios en la Red Municipal de Educación. Cada escuela es responsable de la organización del tiempo escolar, para atender mejor a las demandas de la comunidad local.

2 EJA BEM. Vinculado al BEM-BH y al EJA, atiende a grupos de personas que funcionan en diversos espacios públicos y comunitarios de la ciudad. 
3 Enseñanza Fundamental Nocturna. Esta es regular y debe cumplir el calendario oficial de la Enseñanza Fundamental, que exige 200 días lectivos y 800 horas/clase.

4 Brasil alfabetizado. Programa del Gobierno Federal en colaboración cón el gobierno municipal de Belo Horizonte en la alfabetización de jóvenes y adultos. Los cursos tienen duración de ocho meses, con carga horaria de 320 horas y también funcionan en espacios públicos y comunitarios del municipio.

* Excepto Brasil Alfabetizado, los demás cursos suministran certificados a los alumnos.

\section{Curso de Directores y curso de Coordinadores pedagógicos}

Promovidos por el Centro de Formación de los Profesionales de la Educación-CAPE, estos cursos han sido orientados hacia la formación específica de los profesionales que actúan en la gestión y coordinación de las escuelas. Su objetivo es capacitar a estos profesionales para que enfrenten y articulen los desafíos de la construcción de una escuela democrática, inclusiva y de calidad.

\section{BH para niños}

Este programa educativo creado en 1995 busca ampliar el horizonte de formación de los alumnos al explorar la ciudad como espacio de construc-

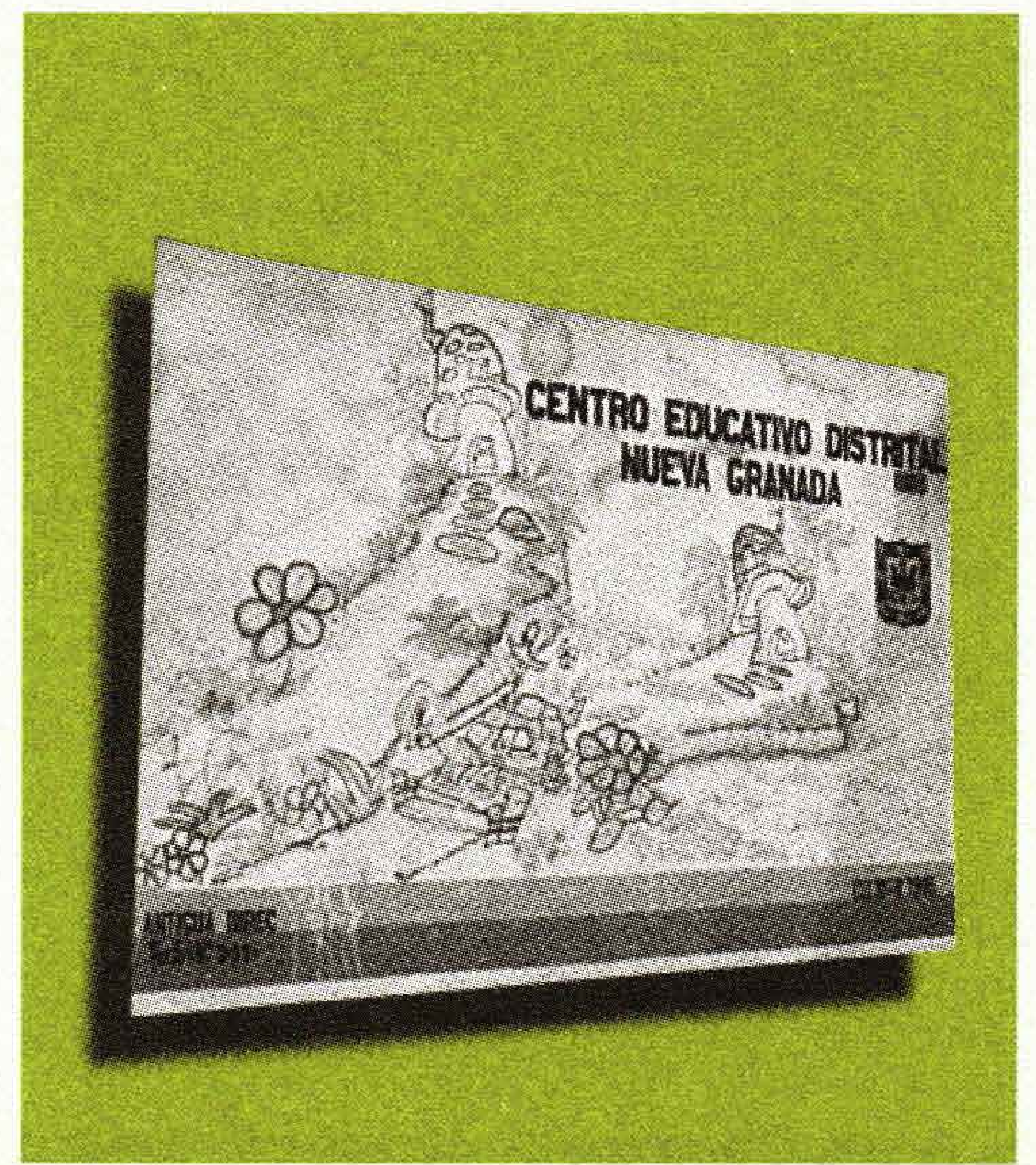

ción de identidades, conocimientos y culturas. Se apropia de la ciudad como espacio de reflexión y de conocimiento.

El programa organiza una diversificada agenda de visitas de alumnos y profesores a museos, teatros, cines, fábricas, galerías de arte, emisoras de radio y televisión, diarios, parques y otros espacios culturales como Red por la paz.

\section{Muestra plural}

Esta se realiza anualmente desde 1995, en espacio público, con el objetivo de presentar a la ciudad los trabajos que profesores y alumnos de la Red Municipal de Educación construyen en sus escuelas. 


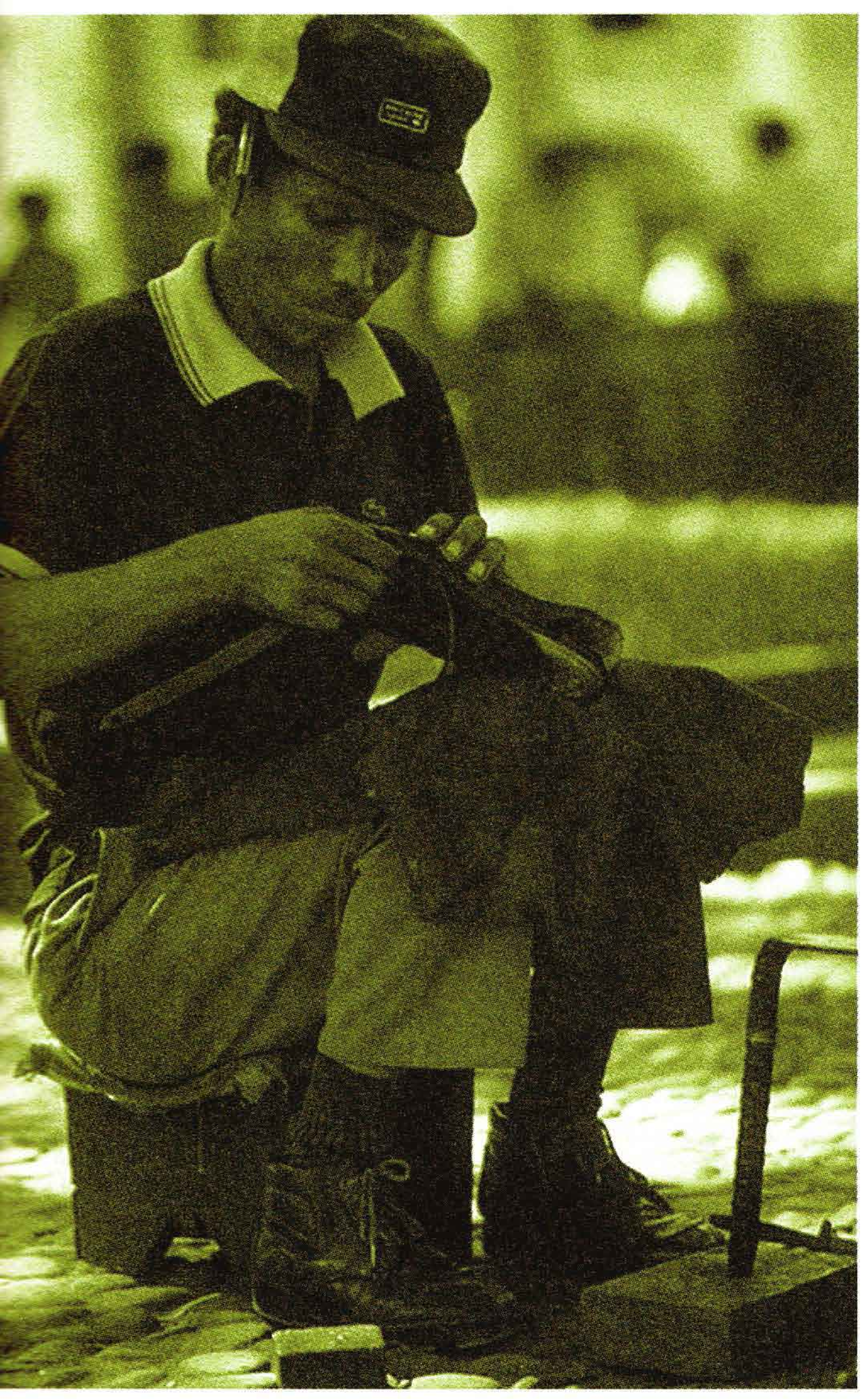

\section{Red por la paz}

Es un programa permanente de prevención de accidentes y violencia en las escuelas. Tiene por objetivo desarrollar una política pedagógica orientada hacia una cultura de paz. Este se viene implementado en todas las escuelas municipales y ofrece cursos para profesores que desarrollan proyectos sobre el tema de la violencia en las escuelas.

Entre las acciones en curso destacamos la implantación de las Comisiones Internas de Prevención a Accidentes y Violencia en las Escuelas, CIPAVE, en cumplimiento a la Ley Municipal 8517/2003.

\section{Tempo integral}

Programa que tiene la finalidad de ampliar el tiempo de permanencia de los alumnos en la escuela, con clases de estímulo a la creatividad.

\section{Escuela Abierta en la Escuela plural}

Este programa consiste en la apertura de las escuelas los fines de semana. Ofrece actividades de recreación, deportes, formación y cultura basadas en los valores de la cultura de paz, educación a la tolerancia, comprensión y respeto a la diferencia. Además de proporcionar una mayor interacción entre la escuela y la comunidad, posibilita estrechar las relaciones entre profesores, alumnos y sus familiares. Lanzado como proyecto piloto, el 30 de octubre de 2004, luego de seis meses de su implantación ya era considerado como referencia para el país y adoptado como modelo por el Ministerio de 
Educación, MEC. Pionero en la capital de Minas Gerais, el Programa será ampliado de las actuales 54 escuelas para otras 50 escuelas de la RME, totalizando 104 espacios abiertos a las comunidades en Belo Horizonte. Escuela Abierta es desarrollado en colaboración con el Gobierno Federảl y es coordinado por la Secretaría Municipal de Educación, junto con la Secretaría Municipal de Deportes y Fundación de Cultura de Belo Horizonte.

\section{Capeonline}

Es un ambiente virtual de formación continuada para los profesionales de la Red Municipal de Educación y comunidad en general. Es un espacio para el debate de la práctica pedagógica, intercambio de experiencias y desarrollo del uso de nuevas tecnologías. Conozca el Capeonline:

www.pbh.gov.br/smed/capeonline

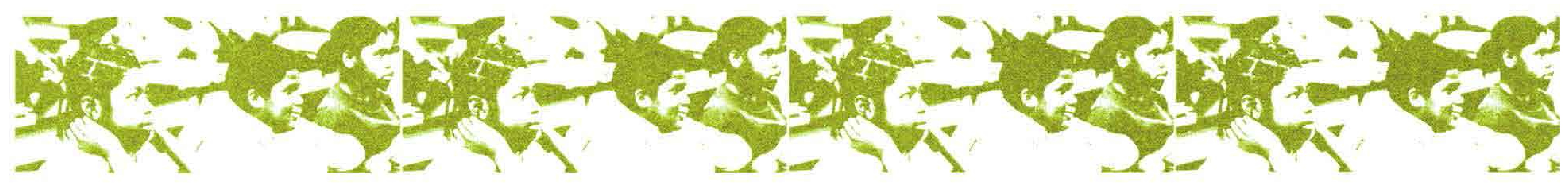





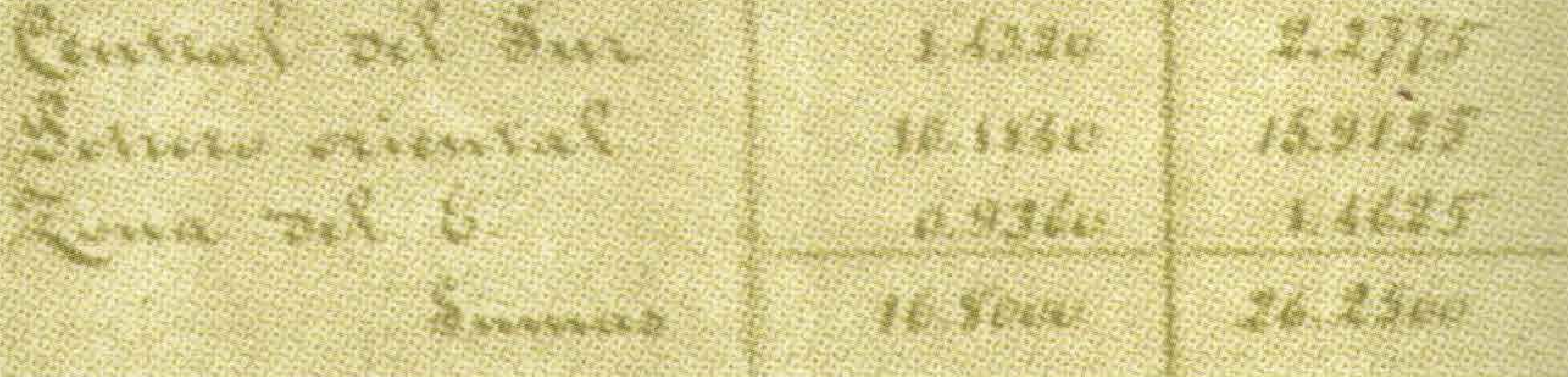

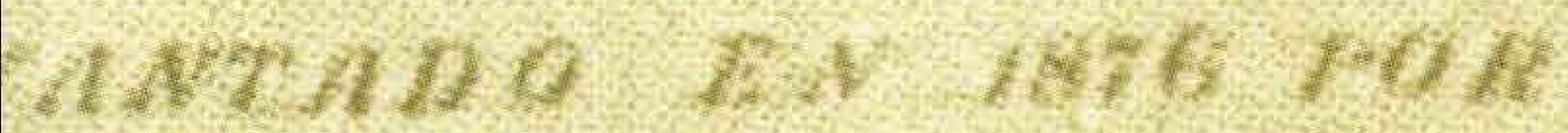

"ypertio Guniva?

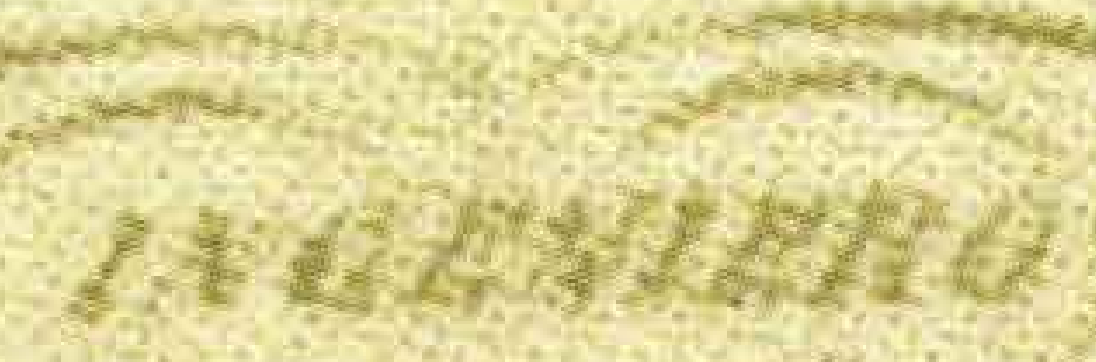

1

$x-2 \leq x$
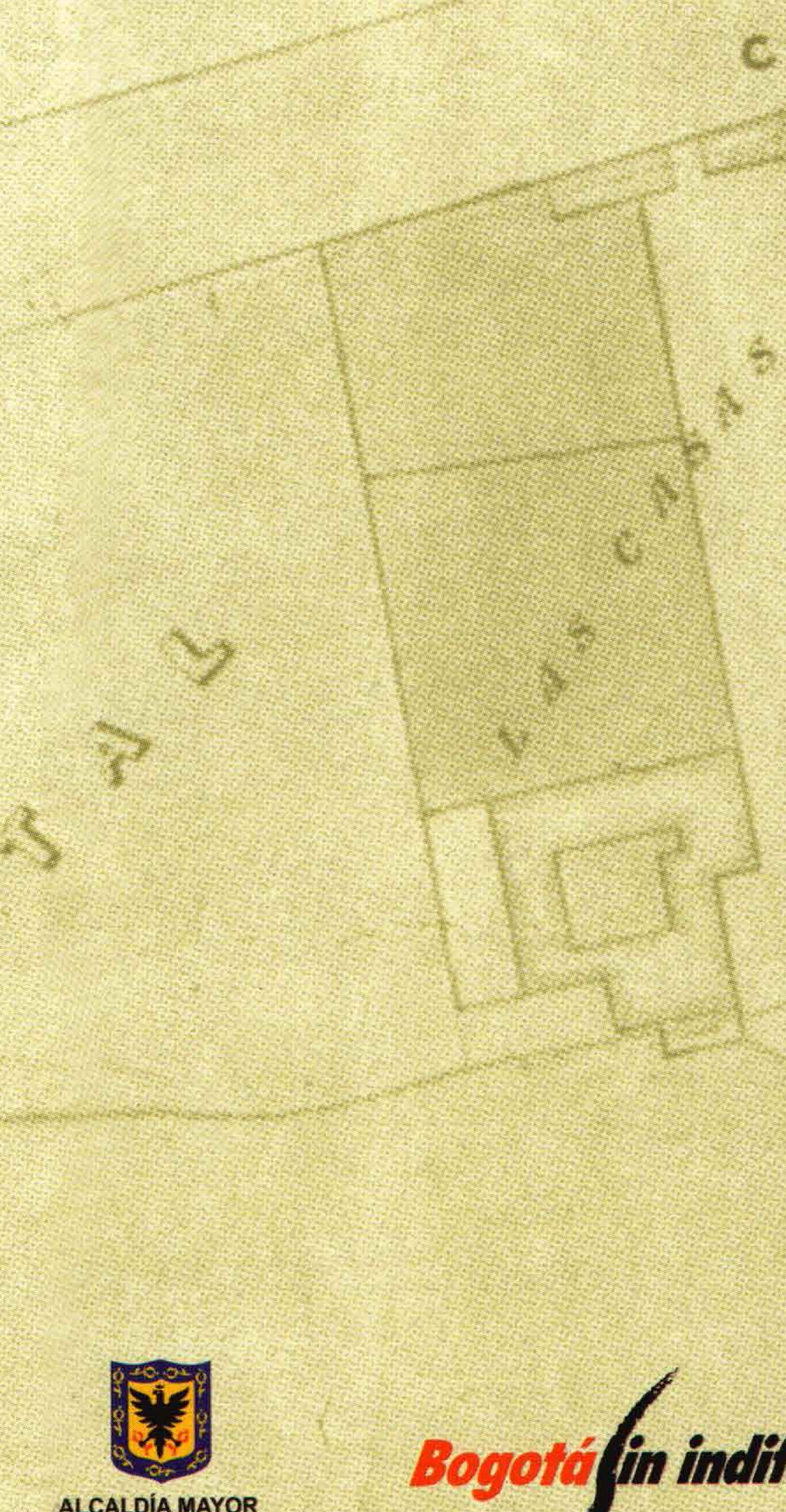
ALCALDIA MAYOR IDEP
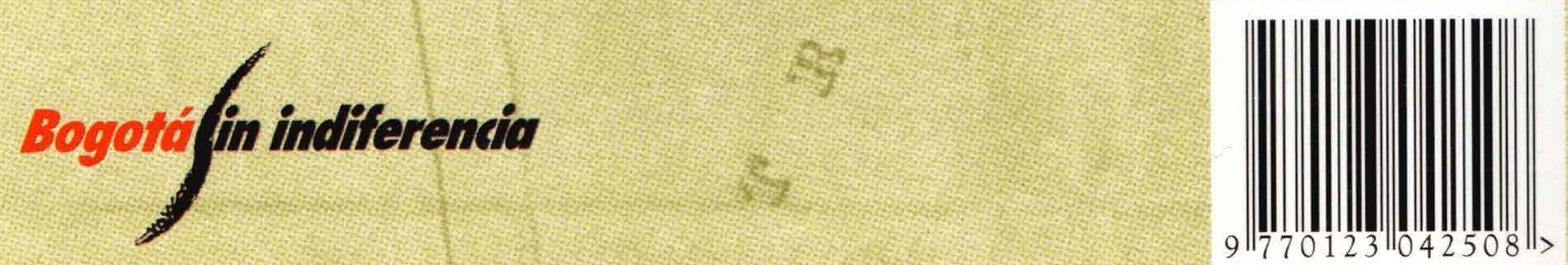